\title{
Weak gravity conjecture constraints on inflation
}

\author{
Qing-Guo Huang \\ School of physics, Korea Institute for Advanced Study, \\ 207-43, Cheongryangri-Dong, Dongdaemun-Gu, \\ Seoul 130-722, Korea \\ huangqg@kias.re.kr
}

\begin{abstract}
We consider the gravitational correction to the coupling of the scalar fields. Weak gravity conjecture says that the gravitational correction to the running of scalar coupling should be less than the contribution from scalar fields. For instance, a new scale $\Lambda=\lambda_{4}^{1 / 2} M_{p}$ sets a UV cutoff on the validity of the effective $\lambda_{4} \phi^{4}$ theory. Furthermore, this conjecture implies a possible constraint on the inflation model, e.g. the chaotic inflation model might be in the swampland.
\end{abstract}


A full quantum theory of gravity has not been formulated. It is a good idea to work out some insights coming from the marriage of gravity and quantum mechanism. Usually we quantize matter field and gravity separately. However, many authors in [1,2] suggested that gravity and the other gauge forces cannot be treated independently. A nontrivial constraint on the really self-consistent gauge theories with gravity appears.

In [2], a new intrinsic UV cutoff for a four-dimensional U(1) gauge theory $\Lambda \sim g M_{p}$ is proposed, where $g$ is the $\mathrm{U}(1)$ gauge coupling and $M_{p}$ is four-dimensional Planck scale. This conjecture is called "weak gravity conjecture" and it provides a possible criterion on the string landscape. However, there are a huge number of meta-stable vacua in string landscape with positive vacuum energy. The authors in [3] proposed a weak gravity conjecture for the case with a cosmological constant. In [4], we showed that it is possible to check this conjecture at the LHC if there are several large extra dimensions and the fundamental Planck scale is roughly $1 \mathrm{TeV}$. This conjecture has also been generalized to higher dimensions $[5,6]$. In particular, the author in [6] gave a new insight on weak gravity conjecture for gauge theories at the one-loop level and suggested a new argument which is independent on monopole and black hole. In [7], the authors proposed a weak gravity conjecture for noncommutative field theories. Other related works are discussed in $[8-15]$.

Guth in [16] suggested a possiblity to solve the horizon, flatness and primordial monopole problem due to a quasi-exponential expansion (inflation) of the universe before hot big bang. However his inflation model called old inflation model leads to an extremely large inhomogeneity and anisotropy of the universe after the phase transition [17]. In $[18,19]$, a new version of inflation to solve previous problems in old inflation model was proposed where inflation is governed by the potential of the scalar fields.

It is well-known that the gauge interaction is determined by symmetry. Unfortunately, there is not a physical principle to constrain the interaction of the scalar fields. In this paper, we generalize the conjecture in [6] to scalar field theories and investigate the constraints on the inflation model.

The interactions between graviton and the scalar fields in $d$ dimensions are described by the action

$$
S=\frac{1}{2 \kappa_{d}^{2}} \int d^{d} x \sqrt{-g} R+\int d^{d} x \sqrt{-g}\left(\frac{1}{2} g^{m n} \partial_{m} \phi \partial_{n} \phi+\frac{1}{2} m^{2} \phi^{2}+V(\phi)\right),
$$

where $\kappa_{d}^{2} \sim G_{d}$ is the $d$-dimensional Newton coupling constant and $V(\phi)$ is the potential of the scalar field. In order to work out the coupling between graviton and scalar field, we consider the quantum fluctuation of the gravitational degrees of freedom around the 
Minkowski metric as

$$
g_{m n}=\eta_{m n}+\kappa_{d} h_{m n} .
$$

The action (11) becomes

$$
S \sim \int d^{d} x(\partial h)^{2}+\int d^{d} x \frac{1}{2} \kappa_{d} \partial_{m} \phi \partial_{n} \phi h^{m n}+\cdots
$$

The interaction term between graviton and the scalar field is proportional to positive powers of $\kappa_{d}$.

In this note we focus on the scalar field theories with polynomial potential. For the potential of the scalar field

$$
V(\phi)=\lambda_{n} \phi^{n},
$$

the scalar field $\phi$ has dimensionality $[\phi]=[\text { mass }]^{\frac{d-2}{2}}$ and the coupling $\lambda_{n}$ takes $\left[\lambda_{n}\right]=$ $[\text { mass }]^{d-\frac{n(d-2)}{2}}$. In general the non-renormalizable interactions are just those whose coupling constants have the dimensionality of negative powers of mass. In $d$ dimensions, $\lambda_{n} \phi^{n}$ term for the scalar field theories are non-renormalizable if $n>\frac{2 d}{d-2}$. For $d=4$, the interaction terms with $n \leq 4$ are renormalizable. In this paper, we only consider the scalar field theories in four dimensions and our results can be easily generalized to higher dimensions. We do not consider three or lower dimensions as gravity does not contain propagating degrees of freedom in these dimensions, even though some of our conjectures may be applicable to these cases.

In [20], Robinson and Wilczek calculated the gravitational correction to running of gauge couplings in four dimensions. In [6], the author gave several examples to support a new viewpoint of weak gravity conjecture which says that the gravitational correction to the $\beta$ function should be less than the contribution from the gauge fields. In this short note, we generalize the observations in [6] to scalar field theories.

First, we consider the scalar field theory with potential

$$
V(\phi)=\lambda_{4} \phi^{4}
$$

The typical Feynman diagrams contributing to the running of the scalar coupling $\lambda_{4}$ are showed in Fig. 1. The dimensional analysis reads the $\beta$ function for $\lambda_{4}$

$$
\beta_{4}=\frac{d \lambda_{4}}{d \ln \Lambda} \sim c_{4} \lambda_{4}^{2}-c_{l} \lambda_{4}\left(\frac{\Lambda}{M_{P}}\right)^{2},
$$

here $c_{4}$ and $c_{l}$ are the numerical coefficients, and $\Lambda$ is an energy scale. When Planck scale is much larger than the scale for the field theory, the gravitational correction can be ignored. Generically the only useful and meaningful notion of the RG running is related 


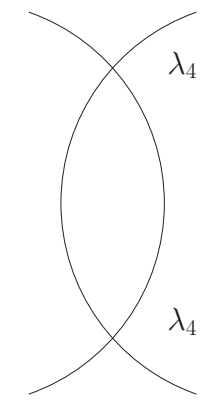

(a)

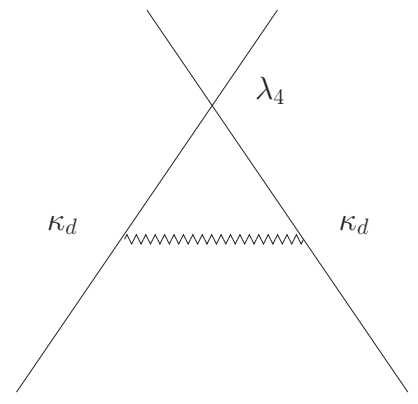

(b)

Figure 1: The typical Feynman diagrams for a scalar field contributing (a) and a gravitational contributing (b) to the $\beta$ function at one-loop level.

to the logarithm corrections to the coupling constant. The power divergence in eq. (6) signals that there is an intrinsic uncertainty in the field theory predictions due to the presence of the higher dimensional operators induced by the gravitational corrections. In principle, we cannot precisely calculate the gravitational corrections before we know the full quantum theory of gravity. We expect that any would-be fundamental theory of quantum gravity should reproduce the same result in the limit of the physical scenario considered here.

The weak gravity conjecture for $\mathrm{U}(1)$ gauge theory is motivated by the absence of the global symmetry for the quantum gravity [2]. Actually this conjecture is consistent with the requirement that the gravity should be the weakest force. This can be also read out from the $\beta$ function [6]. Therefore we generalize this idea to the scalar field theory. Requiring that the contribution from scalar fields is greater than gravitational correction yields

$$
\Lambda^{2} \leq \lambda_{4} M_{P}^{2}
$$

Beyond the scale $\lambda_{4}^{\frac{1}{2}} M_{p}$, the effective $\lambda_{4} \phi^{4}$ theory breaks down and a full quantum theory including gravity is needed. If the mass square is negative, this scalar field plays the role as Higgs field. The vacuum expectation value (vev) of $\phi$ is $\langle\phi\rangle \sim \sqrt{m^{2} / \lambda_{4}}$ which is nothing but the electro-weak scale in standard model. The mass of the physical scalar field around $\langle\phi\rangle$ is still $m$. If the UV cutoff of the scalar field theory $\Lambda$ is smaller than the mass of the scalar field, any quanta of this theory cannot be excited. Naturally $m \leq \Lambda$ is required. Substituting this inequality into eq. (7), we find

$$
\langle\phi\rangle \sim \sqrt{\frac{m^{2}}{\lambda_{4}}} \leq M_{p},
$$

which says that the electro-weak scale should be lower than Planck scale. Eq. (88) is also 
conjectured in [12] where the authors proposed a piece of evidence in two dimensions. It is a reasonable, but trivial observation with the viewpoint of field theory. However there will be a significant implication for inflation model.

Before we consider the weak gravity conjecture constraints on inflation, we investigate another example with potential

$$
V(\phi)=\lambda_{3} \phi^{3}
$$

In principle, we cannot well define this field theory, since its potential has not lower bound. Here we assume that there is still such an effective field theory and the possible Feynman diagrams contributing to the $\beta$ function are showed in Fig. 2. Dimensional analysis reads

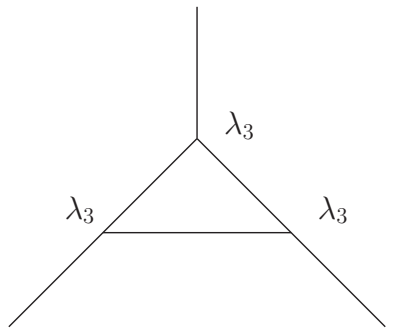

(a)

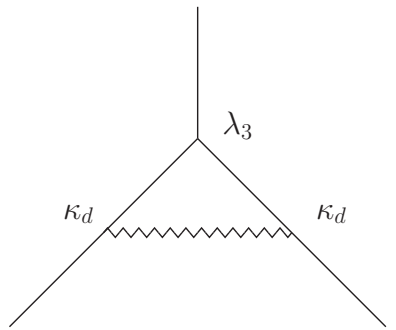

(b)

Figure 2: The typical Feynman diagrams for a scalar field contributing (a) and a gravitational contributing (b) to the $\beta$ function at one-loop level.

that the $\beta$ function takes the form

$$
\beta_{3}=\frac{d \lambda_{3}}{d \ln \Lambda} \sim c_{3} \lambda_{3}^{3} \Lambda^{-2}-c_{l} \lambda_{3}\left(\frac{\Lambda}{M_{P}}\right)^{2}
$$

where $c_{3}$ and $c_{l}$ are the numerical coefficients. Requiring the contribution from scalar field is greater than gravitational correction yields

$$
\Lambda^{2} \leq \lambda_{3} M_{P}
$$

Define a dimensionless coupling $\tilde{\lambda}_{3}=\lambda_{3} / M_{p}$. Eq. (11) is expressed as $\Lambda \leq \tilde{\lambda}_{3}^{1 / 2} M_{p}$.

Following we switch to use the weak gravity conjecture for the scalar field theories to constrain the inflation models. In the slow-roll inflation model, the potential of scalar field provides an effective positive cosmological constant $\rho_{V} \sim V(\phi)$. The Hubble parameter is governed by Friedmann equation

$$
H^{2} \sim \frac{\rho_{V}}{M_{P}^{2}}
$$


Hubble constant acts as the IR cutoff for the field theories. Naturally the IR cutoff of a field theory is lower than the UV cutoff [3], i.e.

$$
H \leq \Lambda
$$

This is nothing but that the size of the universe $H^{-1}$ is larger than the shortest physical length of the field theories $1 / \Lambda$. On the other hand, the quantum fluctuations of the scalar field on this quasi de Sitter background takes the form

$$
\left\langle\phi^{2}\right\rangle \sim H^{2}
$$

Eq. (13) can be interpreted as the quantum fluctuation of the scalar field is smaller than UV cutoff of the field theory.

The evolution of slow-roll inflation is dominated by the potential of scalar field. For instance, we consider the case with $n=4$ in (44). If $\lambda_{4} \phi^{4}$ term dominates the potential, the Hubble constant is related to the scalar field by

$$
H^{2} \sim \lambda_{4} \phi^{4} / M_{P}^{2}
$$

Taking into account the weak gravity conjecture (7) and (13) yields

$$
\frac{\lambda_{4} \phi^{4}}{M_{P}^{2}} \leq \Lambda^{2} \leq \lambda_{4} M_{P}^{2}, \quad \text { or }, \quad \phi \leq M_{P}
$$

The value of scalar field should be smaller than the Planck scale, which is just what we expect. On the other hand, for the case with the evolution of the universe governed by the mass term, i.e. $m^{2} \phi^{2}>\lambda_{4} \phi^{4}$, or,

$$
\phi^{2} \leq \frac{m^{2}}{\lambda_{4}}
$$

the weak gravity conjecture (8) implies $\phi \leq M_{p}$ as well. Similarly, we also get $\phi \leq M_{P}$ for $n=3$. We need to remind the readers that in chaotic inflation model [21] the value of the scalar field is roughly $\sqrt{N} M_{p}$ which is greater than the four-dimensional Planck scale, where $N$ is the number of e-folds before the end of inflation. Weak gravity conjecture implies that chaotic inflation might be in the swampland where the theory is just semiclassically consistent, but not actually inconsistent on the quantum level.

For the scalar field theory with the potential

$$
V(\phi)=V_{0}+\lambda_{4} \phi^{4}
$$

where $V_{0}$ is constant and acts as a zero point energy in field theory. First we ignore the contribution to the $\beta$ function from $V_{0}$. Now the prediction of the weak gravity conjecture 
(17) is still valid. If $\lambda_{4} \phi^{4}$ term dominates the evolution of inflation, the inflation is just $\lambda_{4} \phi^{4}$ inflation and the results have been showed previously. Now we consider the case when the inflation is dominated by $V_{0}$, i.e.

$$
V_{0} \geq \lambda_{4} \phi^{4} .
$$

The Hubble constant takes the form

$$
H^{2} \sim V_{0} / M_{P}^{2} .
$$

Weak gravity conjecture implies that $H^{2} \leq \Lambda^{2} \leq \lambda_{4} M_{P}^{2}$, or

$$
V_{0} \leq \lambda_{4} M_{P}^{4}
$$

Combining with (19), we find $\phi \leq M_{p}$ again. In this inflation model the slow-roll conditions can be satisfied even when the value of the inflaton is smaller than $M_{p}$. But the weak gravity conjecture still brings a constraints on the value of scalar field at the end of inflation $\phi_{e}$, since the total number of e-fold during inflation is greater than 60 at least. The value of the inflaton $\phi_{N}$ at the number of e-folds $N$ before the end of inflation is related to the value of the inflaton at the end of inflation $\phi_{e}$ by

$$
N \sim \frac{V_{0}}{M_{p}^{2} \lambda_{4}}\left(\frac{1}{\phi_{e}^{2}}-\frac{1}{\phi_{N}^{2}}\right) .
$$

This potential of inflaton is a typical potential for hybrid inflation model where the inflation cannot be ended by violating the slow-roll conditions [22]. Combing (22) with (21) implies

$$
\frac{N}{M_{p}^{2}}+\frac{1}{\phi_{N}^{2}} \leq \frac{1}{\phi_{e}^{2}} .
$$

Taking $\phi_{N} \leq M_{p}$ into account, we find

$$
\phi_{e} \leq M_{p} / \sqrt{N+1} .
$$

In order to solve the flatness problem in the hot big bang model, the number of e-folds should not be less than 60 and then $\phi_{e} \leq 0.13 M_{p}$; or, the total number of e-folds has an upper bound $N_{\text {total }} \leq M_{p}^{2} / \phi_{e}^{2}-1$.

We also want to consider the case with potential $V=\lambda_{6} \phi^{6}$. If we only consider $\lambda_{6} \phi^{6}$ term, this theory is non-renormalizable and the $\phi^{4}$ counter term should be naturally included. Usually we don't tune the coefficient of $\phi^{4}$ to be zero in an effective field theory. Therefore we consider the scalar field theory with potential

$$
V(\phi)=\lambda_{4} \phi^{4}+\lambda_{6} \phi^{6} .
$$


The $\beta$ function for $\lambda_{4}$ at the one-loop level takes the form

$$
\beta_{4}=\frac{d \lambda_{4}}{d \ln \Lambda} \sim c_{4} \lambda_{4}^{2}+c_{6} \lambda_{6} \Lambda^{2}-c_{l} \lambda_{4}\left(\frac{\Lambda}{M_{p}}\right)^{2},
$$

where $c_{6}$ is the numerical coefficient and the middle term on the right hand side of (26) comes from Fig. 3. Naively weak gravity conjecture implies that both the first and

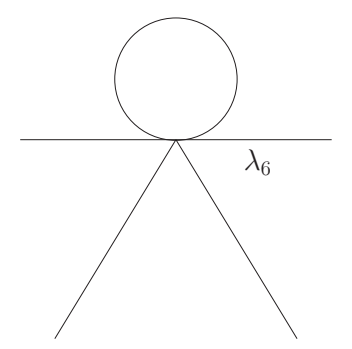

Figure 3: The typical Feynman diagram contributing to $\beta_{4}$ from $\lambda_{6} \phi^{6}$ at one-loop level.

the second term on the right hand side of (26) should be greater than the gravitational correction respectively, i.e.

$$
\frac{\Lambda^{2}}{M_{p}^{2}} \leq \lambda_{4} \leq \lambda_{6} M_{p}^{2}
$$

If $\lambda_{4} \phi^{4}$ term dominates inflation, the results are just the same as the case with only $\lambda_{4} \phi^{4}$. If inflation is driven by $\lambda_{6} \phi^{6}$, the Hubble constant is $H^{2} \sim \lambda_{6} \phi^{6} / M_{p}^{2}$. We rewrite eq. (27) as

$$
\Lambda^{2} \leq \lambda_{6} M_{p}^{4}
$$

This result can be obtained as well from the $\beta$ function for $\lambda_{6}$. Dimensional analysis says that $\beta$ function for $\lambda_{6}$ at the two loop level takes the form $\beta_{6}^{2-l o o p} \sim v_{6} \lambda_{6}^{2} \Lambda^{2}-$ $v_{l} \lambda_{6} \frac{\Lambda^{4}}{M_{p}^{4}}$. Requiring the two-loop gravitational correction is less than the two-loop scalar contribution yields eq. (28). Now $H \leq \Lambda$ also leads to $\phi \leq M_{p}$. This chaotic inflation model might be in the swampland as well.

The previous arguments can be generalized to the cases with multi fields. To be simple, we consider the assisted inflation model [23] with

$$
V\left(\phi_{i}\right)=\lambda_{4} \phi_{i}^{4}, \quad i=1, \ldots, n .
$$

A possible assisted chaotic inflation model in string theory was proposed in [24]. In assisted inflation, there is a unique late-time attractor with all the scalar fields equal, i.e. $\phi_{1}=\phi_{2}=\ldots=\phi_{n}$, where $n$ is the number of scalar fields. With this ansatz, the Hubble constant is given by

$$
H^{2} \sim \frac{n}{M_{p}^{2}}\left(\frac{1}{2} m^{2} \phi^{2}+\lambda_{4} \phi^{4}\right)
$$


where $\phi=\phi_{i}$. The equation of motion for $\phi$ is

$$
3 H \dot{\phi} \simeq-\frac{d V(\phi)}{d \phi} .
$$

The number of e-folds $N$ before the end of inflation is related the value of $\phi_{N}$ by

$$
N \sim \int H d t \sim-\frac{n}{M_{p}^{2}} \int_{\phi_{N}}^{\phi_{e}} \frac{V}{V^{\prime}} d \phi .
$$

Since there is no coupling among these scalar fields from the viewpoint of the field theory, the weak gravity conjecture (17) is still valid for each scalar field $\phi$. If $\lambda_{4} \phi^{4}$ dominates the evolution of inflation, (16) is replaced by

$$
\frac{n \lambda_{4} \phi_{N}^{4}}{M_{p}^{2}} \leq \Lambda^{2} \leq \lambda_{4} M_{p}^{2} .
$$

Using eq. (32), the value of $\phi$ at the number of e-folds $N$ before the end of inflation takes the form $\phi_{N} \sim \sqrt{N / n} M_{p}$. Now eq. (33) reads

$$
N \leq \sqrt{n}
$$

Requiring that the total number of e-folds is not less than 60 implies that the number of inflaton is larger than 3600. On the other hand, the total number of e-folds is bounded by the number of the inflatons. Similar results are also obtained for the case with inflation dominated by $m^{2} \phi^{2}$ term.

To summarize, we propose a weak gravity conjectures for scalar field theories case by case, because there is not a principle to determine the shape of the potential for scalar fields. We only investigate the scalar field theories with polynomial potential and we find a free scalar field theory can not be self-consistent involving gravity. We expect that our results do not rely on perturbation theory, but we usually need to use perturbation theory to calculate the $\beta$ function.

We also use the weak gravity conjecture for the scalar field theories to constrain inflation models. Usually we take the Planck scale as a natural cutoff for the field theories and the field theories do not break down as long as the energy density is smaller than the Planck density. But weak gravity conjecture suggests that gravity cannot be ignored at a new scale lower than Planck scale. As a result, the value of the inflaton should be smaller than Planck scale for chaotic inflation and then we say that chaotic inflation model might not be realized in an effective field theory. Here we need to stress that we cannot give a general argument to support that the value of the inflaton cannot be greater than Planck scale for arbitrary potential. In this paper, we only propose one condition $H \leq \Lambda$ to 
constrain inflation model. More insights are called for if one wants to get more stringent constraints on inflation model. In fact, our results are also valid for quintessence [25], a candidate for dark energy.

Actually our intriguing proposals are still less certain. But we hope that our observations can be taken as the first step on the line to the correct answer.

\section{Acknowledgments}

We would like to thank P.M. Ho, J. Lee, M. Li and F.L. Lin for useful discussions. We also thank department of physics in National Taiwan University for the hospitality. 


\section{References}

[1] C. Vafa, "The string landscape and the swampland," arXiv:hep-th/0509212.

[2] N. Arkani-Hamed, L. Motl, A. Nicolis and C. Vafa, "The string landscape, black holes and gravity as the weakest force," arXiv:hep-th/0601001.

[3] Q. G. Huang, M. Li and W. Song, "Weak gravity conjecture in the asymptotical dS and AdS background," JHEP 0610, 059 (2006) [arXiv:hep-th/0603127].

[4] Q. G. Huang, "Weak Gravity Conjecture with Large Extra Dimensions," arXiv:hep-th/0610106.

[5] T. Banks, M. Johnson and A. Shomer, "A note on gauge theories coupled to gravity," JHEP 0609, 049 (2006) arXiv:hep-th/0606277].

[6] Q. G. Huang, "Gravitational Correction and Weak Gravity Conjecture," JHEP 0703, 053 (2006) arXiv:hep-th/0703039].

[7] Q. G. Huang and J. H. She, "Weak gravity conjecture for noncommutative field theory," JHEP 0612, 014 (2006) arXiv:hep-th/0611211.

[8] S. Kachru, J. McGreevy and P. Svrcek, "Bounds on masses of bulk fields in string compactifications," JHEP 0604, 023 (2006) arXiv:hep-th/0601111.

[9] M. Li, W. Song and T. Wang, "Some low dimensional evidence for the weak gravity conjecture," JHEP 0603, 094 (2006) arXiv:hep-th/0601137.

[10] A. Adams, N. Arkani-Hamed, S. Dubovsky, A. Nicolis and R. Rattazzi, "Causality, analyticity and an IR obstruction to UV completion," JHEP 0610, 014 (2006) arXiv:hep-th/0602178.

[11] H. Ooguri and C. Vafa, "On the geometry of the string landscape and the swampland," arXiv:hep-th/0605264.

[12] M. Li, W. Song, Y. Song and T. Wang, "A weak gravity conjecture for scalar field theories," arXiv:hep-th/0606011.

[13] Y. Kats, L. Motl and M. Padi, "Higher-order corrections to mass-charge relation of extremal black holes," arXiv:hep-th/0606100.

[14] A. J. M. Medved, "An implication of 'gravity as the weakest force'," arXiv:hep-th/0611196.

[15] M. Gasperini, "A new scale in the sky," arXiv:hep-th/0611227. 
[16] A. H. Guth, "The Inflationary Universe: A Possible Solution To The Horizon And Flatness Problems," Phys. Rev. D 23, 347 (1981).

[17] A. H. Guth and E. J. Weinberg, "Could The Universe Have Recovered From A Slow First Order Phase Transition?," Nucl. Phys. B 212, 321 (1983).

[18] A. Albrecht and P. J. Steinhardt, "Cosmology For Grand Unified Theories With Radiatively Induced Symmetry Breaking," Phys. Rev. Lett. 48, 1220 (1982).

[19] A. D. Linde, "Coleman-Weinberg Theory And A New Inflationary Universe Scenario," Phys. Lett. B 114, 431 (1982).

[20] S. P. Robinson and F. Wilczek, "Gravitational correction to running of gauge couplings," Phys. Rev. Lett. 96, 231601 (2006) arXiv:hep-th/0509050.

[21] A. D. Linde, "Chaotic Inflation," Phys. Lett. B 129 (1983) 177.

[22] A. D. Linde, "Hybrid inflation," Phys. Rev. D 49, 748 (1994) arXiv:astro-ph/9307002.

[23] A. R. Liddle, A. Mazumdar and F. E. Schunck, "Assisted inflation," Phys. Rev. D 58, 061301 (1998) arXiv:astro-ph/9804177.

[24] S. Dimopoulos, S. Kachru, J. McGreevy and J. G. Wacker, "N-flation," arXiv:hep-th/0507205.

[25] I. Zlatev, L. M. Wang and P. J. Steinhardt, "Quintessence, Cosmic Coincidence, and the Cosmological Constant," Phys. Rev. Lett. 82, 896 (1999) arXiv:astro-ph/9807002. 